\title{
Inhibition of Rho-associated protein kinase improves the survival of human induced pluripotent stem cell-derived cardiomyocytes after dissociation
}

\author{
MINXIA KE ${ }^{1}$, MENG JI $^{2}, \mathrm{HAO} \mathrm{WANG}^{3}$, YIFENG YAO ${ }^{1}, \mathrm{YUEHONG} \mathrm{WU}^{1}$ and NIANMIN $\mathrm{QI}^{3}$ \\ ${ }^{1}$ Department of Biochemistry and Molecular Biology, College of Life Science and Medicine, \\ Zhejiang Sci-Tech University; ${ }^{2}$ Hangzhou Biaomo Biosciences Co., Ltd., Hangzhou, Zhejiang 310018 ; \\ ${ }^{3}$ Shanghai Likun Biosciences Co., Ltd., Shanghai 201499, P.R. China
}

Received January 9, 2019; Accepted October 8, 2019

DOI: $10.3892 /$ etm.2020.8436

\begin{abstract}
Heart disease remains the leading cause of morbidity and mortality worldwide. Induced pluripotent stem cells (iPSCs) have the ability to differentiate into cardiomyocytes (CMs), rendering this cell type to be a promising pre-cursor of cardiomyocytes for cell-based cardiac regeneration. Obtaining CMs with a high yield and purity coupled with improved subsequent survival could prove to be invaluable for the future cell replacement therapeutic strategies. Rho-associated protein kinase (ROCK) is involved in a wide range of fundamental cellular functions and serves significant roles in cardiac physiology. In the present study, human (h)iPSC-CMs were generated from iPSCs by including glycogen synthase kinase $3 \beta$ and Wnt inhibitors in the basal culture media. The possible effect of Y27632, a ROCK inhibitor, on hiPSC-CMs was then investigated. hiPSC-CMs of high purity were harvested with $>96 \%$ of cells expressing cardiac troponin T. Additionally, treatment with $10 \mu \mathrm{M}$ Y27632 significantly improved the viability of dissociated hiPSC-CMs. The effects of ROCK inhibitors Y27632 and fasudil, on the proliferation and apoptosis of hiPSC-CMs were also examined. Treatment with ROCK inhibitors markedly enhanced hiPSC-CM proliferation, by up to 2.5-fold, whilst Y27632 treatment reduced apoptosis in hiPSC-derived CMs under serum starvation and suspension by suppressing the expression of caspase-3. Taken together, data from the present study indicated that ROCK
\end{abstract}

Correspondence to: Dr Yuehong Wu, Department of Biochemistry and Molecular Biology, College of Life Science and Medicine, Zhejiang Sci-Tech University, 928 Second Avenue, Hangzhou, Zhejiang 310018, P.R. China

E-mail: wuyuehong2003@163.com

Dr Nianmin Qi, Shanghai Likun Biosciences Co., Ltd., 17 Wangyuan Street, Fengxian, Shanghai 201499, P.R. China

E-mail: profqi@vip.163.com

Key words: induced pluripotent stem cells, cardiomyocyte, rho-associated protein kinase inhibitors, Y27632 kinase inhibitors effectively improved the cultural system of hiPSC-derived CMs.

\section{Introduction}

Cardiovascular diseases, including heart failure, chronic ischemic heart disease and acute myocardial infarction, remains a leading cause of morbidity and mortality worldwide (1). The recent advent of the human induced pluripotent stem cell (hiPSC) technology and an increasingly refined capacity to differentiate hiPSCs into cardiomyocytes (CMs), provides an unprecedented opportunity for potential applications in disease modeling, drug discovery, toxicity screening and novel cell-based therapies (2). Over the last two decades, a number of protocols for cardiac differentiation have been established based on embryonic body (EB) and monolayer methodologies (3-5). Obtaining CMs of a high yield and purity by improving cell survival may be invaluable for future applications in cell replacement therapy and novel drug screening.

Rho-associated coiled-coil containing kinases (ROCKs) are members of the serine/threonine protein kinase family that are well documented to be involved in the regulation of a wide range of fundamental cellular functions, including cell proliferation, apoptosis, cell mitosis adhesion, cytoskeletal adjustments and muscle cell contraction $(6,7)$. Evidence from a plethora of animal and clinical studies have suggested ROCKs to be important mediators of a number of pathophysiological characteristics associated with cardiovascular diseases, including cardiac remodeling, fibrosis and hypertrophy $(6,8)$. Of note, Y27632, a pharmacological inhibitor of ROCK and the calcium-sensitization pathway of smooth muscle contraction, has been previously applied as a therapeutic drug for the treatment of cardiovascular diseases (9). Apart from Y27632, fasudil is another potent ROCK inhibitor and vasodilator which has been used for the treatment of cerebral vasospasm (10). Indeed, a number of previous studies have indicated that ROCK 1 and ROCK2 contribute to pathological CM apoptosis $(11,12)$. Additionally, long-term fasudil and Y27632 treatment has been demonstrated to attenuate CM apoptosis and limit the progression of pathological cardiac fibrosis, hypertrophy and remodeling, which lead to heart 
failure $(13,14)$. However, the effects of the ROCK signaling pathway on hiPSC-derived CMs have not yet been thoroughly investigated.

Therefore, the aim of the present study was to examine whether ROCK inhibitors can be used to increase the survival of hiPSC-derived CMs, which may facilitate the development of applications in stem cell therapy for the treatment of heart disease. Specifically, the potential effects of the ROCK inhibitors, Y27632 and fasudil, were evaluated on hiPSC-derived CMs. The results indicated that ROCK inhibitors promoted hiPSC-CM proliferation, increased the number of viable cells and inhibited the apoptosis of hiPSC-CMs in suspension. The mechanism of these physiological effects mediated by the ROCK inhibitor on hiPSC-CM apoptosis may be via the suppression of caspase-3 expression.

\section{Materials and methods}

The differentiation of hiPSC into cardiomyocytes using small molecules. HiPSCs (cat. no. DYR0100 Type Culture Collection of the Chinese Academy of Sciences) were cultured at $37^{\circ} \mathrm{C}$ and $5 \% \mathrm{CO}_{2}$ in $\mathrm{mTeSR}^{\mathrm{TM}} 1$ maintenance medium (Stemcell Technologies, Inc.). Cells were seeded into $35 \mathrm{~mm}$ culture dishes $\left(2 \times 10^{5}\right.$ cells/dish) coated with Matrigel (Corning, Inc.) and passaged once every 2-3 days using ACCUTASE ${ }^{\mathrm{TM}}$ cell detachment solution (Stemcell Technologies, Inc.).

For direct differentiation into cardiomyocytes (5), hiPSC first were split at a 1:6 ratio using mTeSR media and cultured for 2 days to $\sim 85 \%$ confluence. On day 0 , cells were first treated with $6 \mu$ M GSK- $\beta$ inhibitor CHIR99021 (MedChemExpress) diluted in in RPMI/B27 without insulin for $48 \mathrm{~h}$, followed by transferal to RPMI/B27 minus insulin supplemented with $3 \mu \mathrm{g} / \mathrm{ml}$ Wnt signaling inhibitor IWP2 (MedChemExpress) for another 3 days. On day 5 following CHIR99021 treatment, the medium was changed to RPMI/B27 without insulin and finally to RPMI/B27 with insulin medium on day 7 for further maintenance until day 21.

To purify hiPSC-CMs, a biochemical purification protocol was used using glucose-depleted DMEM (Invitrogen; Thermo Fisher Scientific, Inc.) supplemented with $4 \mathrm{mM}$ lactate (Sigma-Aldrich; Merck KGaA) on day 15 (15). On day 15, the medium was changed to the purification medium, consisting of glucose depleted DMEM, $500 \mu \mathrm{g} / \mathrm{ml}$ recombinant human albumin (Oryzogen; Wuhan Healthgen Biotechnology, Corp.) and $213 \mu \mathrm{g} / \mathrm{ml} \mathrm{l-ascorbic} \mathrm{acid} \mathrm{2-phosphate} \mathrm{supplemented}$ (Sigma-Aldrich; Merck KGaA) with $4 \mathrm{mM}$ 1-lactic acid (Sigma-Aldrich; Merck KGaA). Medium was refreshed every 2 days during the purification process. On day 19, cells were maintained in RPMI/B27 media and allowed to recover for 2 days.

To investigate the effects of Y27632 on hiPSC-CMs, cells were first dissociated and seeded into gelatin-coated 12-well plates (Corning, Inc.) at a density of $3 \times 10^{5}$ cells/well under a number of different conditions. For controls, hiPSC-CMs were cultured in DMEM supplemented with 20\% FBS (Invitrogen; Thermo Fisher Scientific, Inc.) in the absence of Y27632 (Selleck Chemicals). For other treatment groups, cells were cultured in DMEM containing 20\% FBS supplemented with various concentrations of Y27632 $(5,10$ or $20 \mu \mathrm{M})$. After 24 and $48 \mathrm{~h}$, cells were washed in PBS, trypsinized and counted $(\mathrm{n}=3)$. Specifically, $10 \mu \mathrm{l}$ cells mixed with equal volumes of $0.4 \%$ trypan blue dye (Beijing Solarbio Science \& Technology Co., Ltd.) were counted using a hemocytometer.

Immunostaining and fluorescent microscopy. Cells were first fixed in 4\% paraformaldehyde (Beyotime Institute of Biotechnology) for $15 \mathrm{~min}$ at room temperature (RT), permeabilized with $0.1 \%$ Triton X-100 (Sigma-Aldrich: Merck KGaA) for 10 min at RT and blocked with $10 \%$ goat serum (Sigma-Aldrich; Merck KGaA) at RT for $1 \mathrm{~h}$. Samples were subsequently stained with primary antibodies against cardiac troponin $\mathrm{T}$ (cTnT; 1:200; cat. no. 710635; Invitrogen; Thermo Fisher Scientific, Inc.) and $\alpha$-actinin (Invitrogen; 1:200; cat. no. 710947; Thermo Fisher Scientific, Inc.), dissolved in PBS at $4^{\circ} \mathrm{C}$ for $12 \mathrm{~h}$ and further incubated with Alexa Fluor ${ }^{\mathrm{TM}}$ 555-conjugated antibody (1:500; cat. no. A0460; Beyotime Institute of Biotechnology) and Alexa Fluor ${ }^{\mathrm{TM}}$ 488-conjugated antibody (1:500; cat. no. A0423; Beyotime Institute of Biotechnology) at RT for $2 \mathrm{~h}$. Nuclei were stained using $1 \mu \mathrm{g} / \mathrm{ml}$ DAPI (Sigma-Aldrich; Merck KGaA) at RT for $5 \mathrm{~min}$. An Optiphot-2 microscope (Nikon Corporation) equipped with a CCD video camera system (Optronics Engineering, Ltd.) and a computer interface (NIS-Elements; version 4.2.0; Nikon Corporation) was used for imaging analysis (magnification, x100). In total, 40 images were taken for each well.

Crystal violet staining. Cells were first washed with PBS, fixed in $4 \%$ paraformaldehyde at RT for 15 min and stained with $0.1 \%$ crystal violet solution (Sigma-Aldrich; Merck $\mathrm{KGaA}$ ) at RT for $30 \mathrm{~min}$. After the cells were washed with $\mathrm{ddH}_{2} \mathrm{O}$ and air-dried at RT, images were obtained using a color digital camera (Optronics Engineering, Ltd.). Absorbance was subsequently measured at $590 \mathrm{~nm}$ using an automatic microplate reader (BioTek ${ }^{\mathrm{TM}} \mathrm{ELx}_{800}^{\mathrm{TM}}$; Omega Bio-Tek, Inc.).

Cell counting kit-8 (CCK-8) assay. Cells were plated into 96-well plates in triplicate at $\sim 10,000$ cells/well and subsequently cultured with or without $10 \mu \mathrm{M} \mathrm{Y} 27632$ at $37^{\circ} \mathrm{C}$ and $5 \% \mathrm{CO}_{2}$ for $24 \mathrm{~h}$. Cells were analyzed using CCK-8 assay according to the manufacturer's protocol (Beyotime Institute of Biotechnology). In total, $10 \mu \mathrm{l}$ CCK-8 were added per well and incubated for $2 \mathrm{~h}$ at $37^{\circ} \mathrm{C}$. The absorbance value at a wavelength of $450 \mathrm{~nm}$ was measured for each well using an enzyme-linked immunosorbent assay reader (Thermo Lab Systems; Thermo Fisher Scientific, Inc.).

5-bromo-2-deoxyuridine (BrdU) incorporation assay. hiPSC-CM proliferation was evaluated using BrdU incorporation assay. The same number of hiPSC-CMs $\left(1.5 \times 10^{5}\right.$ cells $)$ were cultured in DMEM supplemented with $20 \%$ FBS and $10 \mu \mathrm{M} \mathrm{Y} 27632$ for $24 \mathrm{~h}$ after passage on day 21, following which the cells were treated with or without $10 \mu \mathrm{M}$ Y27632 for 3 or 6 days, and incubation with or without $10 \mu \mathrm{M}$ fasudil for 6 days (16). Cells were then treated with $10 \mu \mathrm{M} \mathrm{BrdU}$ (Sigma-Aldrich; Merck KGaA) and incubated at $37^{\circ} \mathrm{C}$ for $6 \mathrm{~h}$. Samples were then fixed using $4 \%$ paraformaldehyde at RT for $15 \mathrm{~min}$, permeabilized by $0.1 \%$ Triton X-100 at RT for $15 \mathrm{~min}$ and acid-washed by $1 \mathrm{M} \mathrm{HCl}$ on ice for $10 \mathrm{~min}$. BrdU-positive cells were subsequently detected by immunocytochemistry using mouse anti-BrdU (1:1,000; 
cat. no. B2531; EMD Millipore) at $4{ }^{\circ} \mathrm{C}$ overnight and goat anti-mouse FITC-conjugated secondary antibodies (1:400; cat. no. BA1101; Molecular Probes; Thermo fisher Scientific, Inc.) at RT for $1 \mathrm{~h}$. Nuclei were visualized via $1 \mu \mathrm{g} / \mathrm{ml}$ DAPI staining at RT for $5 \mathrm{~min}$. In total, each dish was divided into 4 regions, where 10 images were taken per region; 2,000 cells were quantified and normalized to the total cell number in each field using ImageJ software (version 1.8.0; National Institutes of Health).

Annexin V-FITC/propidium iodide (PI) apoptosis detection by fluorescence-activated cell sorting (FACS) assay. Cell apoptosis was assessed using annexin-V-FITC and PI staining kit (BD Biosciences). In total, $5 \times 10^{5}$ cells were collected by trypsinization at $37^{\circ} \mathrm{C}$ for $5 \mathrm{~min}$, centrifuged under $200 \mathrm{x}$ g at $4^{\circ} \mathrm{C}$ for $5 \mathrm{~min}$ and washed twice with PBS, resuspended in $500 \mu \mathrm{l}$ $1 \mathrm{X}$ binding buffer and incubated in $5 \mu \mathrm{l}$ Annexin V-FITC and $5 \mu \mathrm{l}$ PI solution in the dark for $15 \mathrm{~min}$ at RT. Cells were analyzed using an integrated BD Accuri ${ }^{\mathrm{TM}}$ C6 system (BD Biosciences). The gates for flow cytometry was set using following steps: Target cell population was circled by detecting unstained cells. Annexin V-FITC single positive cells were detected, following which fluorescence compensation was adjusted to ensure no particles were in the upper left (UL) and upper right (UR) quadrants. PI single positive cells were detected and fluorescence compensation was adjusted to ensure no particles were present in the upper right (UR) and lower right (LR) quadrants. A total of four populations of cells were observed: i) Cells that were viable and not undergoing apoptosis are both FITC-Annexin V and PI-negative (LL); ii) cells undergoing apoptosis were FITC-Annexin V-positive and PI-negative (LR); iii) the cells that are undergoing late apoptosis or necrotic are both positive for FITC-Annexin V and PI (UL and UR).

Reverse transcription-quantitative PCR (RT-qPCR) or semi-quantitative PCR analysis ( $R T-P C R)$. Total RNA was extracted and prepared using TRIzol ${ }^{\circledR}$ reagent (Invitrogen; Thermo Fisher Scientific, Inc.) according to the manufacturer's protocol. cDNA was synthesized from $1 \mu \mathrm{g}$ RNA using the AccuPower ${ }^{\circledR}$ RT Premix (Bioneer Corporation). The temperature for the reverse transcription reaction was $25^{\circ} \mathrm{C}$ for $5 \mathrm{~min}, 42^{\circ} \mathrm{C}$ for $60 \mathrm{~min}$ and $70^{\circ} \mathrm{C}$ for $5 \mathrm{~min}$, following which the product was used for RT-qPCR and RT-PCR analyses.

RT-PCR was performed using ACTB mRNA as the normalizing internal control and carried out using Taq DNA Polymerase (Takara Bio, Inc.). The thermocycling conditions (total volume per reaction, $20 \mu \mathrm{l}$ ) were as follows: Initial $5 \mathrm{~min}$ denaturation at $95^{\circ} \mathrm{C}$, followed by 30 cycles of $95^{\circ} \mathrm{C}$ for $30 \mathrm{sec}$, $60^{\circ} \mathrm{C}$ for $30 \mathrm{sec}$ and $72^{\circ} \mathrm{C}$ for $30 \mathrm{sec}$ and a final extension for $10 \mathrm{~min}$ at $72^{\circ} \mathrm{C}$. PCR products were resolved by electrophoresis on a $1.5 \%$ agarose gel and stained using GelRed ${ }^{\circledR}$ Nucleic acid gel stain (Biotium, Inc.), then visualized via UV transillumination and photographed using a Gel Doc ${ }^{\mathrm{TM}}$ EZ System (Bio-Rad Laboratories, Inc.).

qPCR reactions were set up in duplicate using $\mathrm{SYBR}^{\circledR}$ Premix Ex Taq ${ }^{\mathrm{TM}}$ (Takara Bio, Inc.) and performed using the 7500 Real-Time PCR system (Applied Biosystems; Thermo Fisher Scientific, Inc.). qPCR reactions of $20 \mu \mathrm{l}$ for eachsample
Table I. Primers used for PCR for the present study.

\begin{tabular}{|c|c|}
\hline Genes & Sequence $\left(5^{\prime}-3^{\prime}\right)$ \\
\hline ACTB & $\begin{array}{l}\text { F: AGCGAGCATCCCCCAAAGTT } \\
\text { R: GGGCACGAAGGCTCATCATT }\end{array}$ \\
\hline TNNT2 & $\begin{array}{l}\text { F: TTCACCAAAGATCTGCTCCTC } \\
\text { GCT } \\
\text { R: TTATTACTGGTGTGGAGTGGGTG } \\
\text { TGG }\end{array}$ \\
\hline TNNI3 & $\begin{array}{l}\text { F: CTGCAGATTGCAAAGCAAGA } \\
\text { R: CCTCCTTCTTCACCTGCTTG }\end{array}$ \\
\hline MYL7 & $\begin{array}{l}\text { F: GAGGAGAATGGCCAGCAGGAA } \\
\text { R: GCGAACATCTGCTCCACCTCA }\end{array}$ \\
\hline MYL2 & $\begin{array}{l}\text { F: ACATCATCACCCACGGAGAAG } \\
\text { AGA } \\
\text { R: ATTGGAACATGGCCTCTGGAT } \\
\text { GGA }\end{array}$ \\
\hline Bax & $\begin{array}{l}\text { F: CCCCCGAGAGGTCTTTTTCC } \\
\text { R: TGTCCAGCCCATGATGGTTC }\end{array}$ \\
\hline $\mathrm{Bcl}-2$ & $\begin{array}{l}\text { F: GAACTGGGGGAGGATTGTGG } \\
\text { R: AAAGCCAGCCTCCGTTATCC }\end{array}$ \\
\hline Caspase-3 & $\begin{array}{l}\text { F: GGGGATTGTTGTAGAAGTCTA } \\
\text { ACT } \\
\text { R: AATAACCAGGTGCTGTGGAGTA }\end{array}$ \\
\hline Caspase- 8 & $\begin{array}{l}\text { F: ATTAGGGACAGGAATGGAACAC } \\
\text { R: GGAGAGGATACAGCAGATGAAG }\end{array}$ \\
\hline
\end{tabular}

ACTB, $\beta$-actin; TNNT2, troponin $\mathrm{T}$ type 2 ; TNNI3, troponin I type 3; MYL7, myosin light chain 7; MYL2, Myosin light chain 2; $\mathrm{F}$, forward; R, reverse.

were made and consisted of cDNA (200 $\mu \mathrm{g}$ cDNA after dilution), 2X SYBR Premix Ex Taq, 50X ROX Reference Dye, $\mathrm{dH}_{2} \mathrm{O}$ and $10 \mu \mathrm{M}$ of each gene-specific primer. The thermocycling conditions were as follows: Initial denaturation at $95^{\circ} \mathrm{C}$ for $30 \mathrm{sec}$, followed by 40 cycles of $95^{\circ} \mathrm{C}$ for $5 \mathrm{sec}$, annealing at $60^{\circ} \mathrm{C}$ for $34 \mathrm{sec}, 72^{\circ} \mathrm{C}$ for $30 \mathrm{sec}$ and a final extension at $72^{\circ} \mathrm{C}$ for $10 \mathrm{~min}$. Relative mRNA abundance was calculated using the $2^{-\Delta \Delta \mathrm{Cq}}$ method (17) and normalized to the expression levels of ACTB. The sequences of the primers used are listed in Table I.

Western blot analysis. Cells were prepared and lysed in ice-cold RIPA buffer (0.5 M Tris- $\mathrm{HCl}, \mathrm{pH} 7.4,1.5 \mathrm{M} \mathrm{NaCl}$, $2.5 \%$ deoxycholic acid, $10 \% \mathrm{NP}-40$ and $10 \mathrm{mM}$ EDTA; EMD Millipore) containing freshly added Halt Phosphatase Inhibitor Cocktail (Thermo Fisher Scientific, Inc.) and Protease Inhibitor Cocktail (Sigma-Aldrich; Merck KGaA). Protein concentrations were determined using Bradford protein assay (Bio-Rad Laboratories, Inc.). In total, $20 \mu \mathrm{g}$ protein samples were separated by $10 \%$ or $12 \%$ SDS-PAGE using an electrophoresis system (Bio-Rad Laboratories, Inc.) and transferred onto nitrocellulose membranes. The membranes were subsequently 
blocked with $5 \%$ non-fat milk for $1 \mathrm{~h}$ at RT and probed with a number of different primary antibodies (GAPDH, cat. no. $2118 \mathrm{~S}$; cleaved caspase-3, cat. no. $9661 \mathrm{~S}$; or caspase-8 cat. no. 4790S; all 1:1,000; Cell Signaling Technology, Inc.) at $4^{\circ} \mathrm{C}$ overnight. Samples were then incubated with horseradish peroxidase-conjugated goat anti-rabbit $\operatorname{IgG}(\mathrm{H}+\mathrm{L})$ (1:2,500; cat. no. SA00001-2; Proteintech Group, Inc.) at RT for $2 \mathrm{~h}$. Blots were visualized using enhanced chemiluminescence (ECL) reagent (Amersham; GE Healthcare Life Sciences), images were captured using the ECL Tanon 5500 system (Tanon Science and Technology Co., Ltd.) and analyzed using Tanon 5500 Multi automatic chemiluminescence/fluorescent image analysis system (Tanon Science and Technology Co., Ltd.).

FACS analysis. Following purification, $5 \times 10^{5}$ iPSC-CMs were trypsinized under $37^{\circ} \mathrm{C}$ for $5 \mathrm{~min}$, centrifugated at $200 \mathrm{x} \mathrm{g}$ at RT for $5 \mathrm{~min}$, fixed and permeabilized with $70 \%$ ethanol at $4^{\circ} \mathrm{C}$ overnight and stained with phycoerythyrin-conjugated mouse anti-cTnT antibodies (1:200; cat. no. 564767; BD Biosciences; for $2 \mathrm{~h}$ at $4^{\circ} \mathrm{C}$. Cells were washed three times with PBS and analyzed using flow cytometry (BD Accuri ${ }^{\mathrm{TM}}$ C6, BD Biosciences).

Analysis of caspase-3 activity. Caspase-3 activity was detected using a caspase-3 Activity Assay kit (cat. no. C1116, Beyotime Institute of Biotechnology). Cells $\left(5 \times 10^{5}\right)$ were cultured in the presence or absence of $10 \mu \mathrm{M}$ Y 27632 for 3 days in a 6-well plate, which were then washed with PBS, resuspended in the RIPA lysis buffer (as part of the caspase-3 Activity Assay kit) and incubated on ice for $15 \mathrm{~min}$. For each well of a 96-well microplate, cell lysates (50 $\mu \mathrm{l} /$ well), assay buffer and caspase-3 substrate (Ac-DEVD-pNA, $10 \mu \mathrm{l} /$ well) were combined together. Samples were then incubated at $37^{\circ} \mathrm{C}$ for $2 \mathrm{~h}$ and quantified using microplate reader by measuring absorbance at $405 \mathrm{~nm}$.

Statistical analysis. All experiments of the current study were repeated three times and the data are presented as the mean \pm standard deviation. GraphPad Prism 7 (GraphPad Software, Inc.) software was used for the statistical analysis of all data. Statistical differences between two groups were analyzed using Student's t-test (two-tailed unpaired). Comparisons between multiple groups were performed using one-way ANOVA followed by a Dunnett's post hoc multiple comparison test. $\mathrm{P}<0.05$ was considered to indicate a statistically significant difference. To detect caspase-3 activity, a linear regression line (Microsoft Corporation) was fitted to the concentration: Optical density data. For comparative purposes, caspase-3 activity values were generated for each group using regression analyses.

\section{Results}

Cardiac differentiation and characteristics of hiPSC-CMs. hiPSC-CMs were generated from hiPSC using CHIR9901 and IWP-2, inhibitors of GSK3 $\beta$ and Wnt respectively, in insulin-free medium (Fig. 1A) (5). Following $~ 8$ days after CHIR99021 treatment, spontaneously contracting cells were observed. As presented in Fig. 1B, the expression of cardiac markers, including troponin T type 2 (TNNT2), troponin I type 3 (TNNI3), MYL7 and MYL2, were revealed to be positive by RT-PCR analysis. In addition, hiPSC-CMs displayed positive expression for cTnT and sarcomeric- $\alpha$ actinin on day 15 (Fig. 1C). FACS analysis demonstrated that the purity of hiPSC-CMs isolated appeared to be high, with $>96 \%$ cells expressing cTnT on day 21 (Fig. 1D).

ROCK inhibitor markedly promotes the survival of dissociated of hiPSC-CMs. In the present study, the potential effects of Y27632, a ROCK inhibitor, were investigated on hiPSC-CMs. To examine if Y27632 exerts an effect on hiPSC-CM viability, cells were cultured in the presence of $0,5,10$ or $20 \mu \mathrm{M}$ Y27632 for $24 \mathrm{~h}$. The number of viable cells increased in a dose-dependent manner, as the concentration of Y27632 increased to 5 and $10 \mu \mathrm{M}$ (Fig. 2A). However, at $20 \mu \mathrm{M}$, the number of viable cells was decreased compared with $10 \mu \mathrm{M}$ (Fig. 2A). Subsequently, the effects of Y27632 on the viability of hiPSC-CMs, quantified as the number of cells positively staining for the TNNT2, were examined. Y27632 $(10 \mu \mathrm{M})$ markedly increased the plating efficiency of hiPSC-CMs (Fig. 2B). The images and values obtained from crystal violet staining demonstrated a significant increase in relative cell viability following $10 \mu \mathrm{M}$ Y27632 treatment compared with untreated cells (Fig. 2C). Quantitative analysis of the data obtained from the CCK-8 assay also reported significant increases in relative cell viability in Y27632 treated cells compared with untreated cells (Fig. 2D). Taken together, these data indicated that Y27632 treatment enhanced the viability of hiPSC-CMs.

ROCK inhibitor enhances hiPSC-CMs proliferation. To determine whether the increased cell viability was also due to increased cell proliferation, a BrdU incorporation assay coupled with fluorescent microscopy was performed to measure hiPSC-CM proliferation. After cells were cultured for 3 or 6 days in the absence or presence of $10 \mu \mathrm{M} \mathrm{Y} 27632$, fluorescence images were taken on day 3 (Fig. 3A) and day 6 (Fig. 3B). Significant increases in the numbers of BrdU-positive cells were observed following Y27632 treatment for both days $(\mathrm{P}<0.0001$; Fig. 3A and B). On day 3, the mean number of $\mathrm{BrdU}^{+}$cells counted in fluorescent images were $2.12 \pm 0.02$ and $4.88 \pm 0.07 \%$ (Fig. $3 \mathrm{~A} ; \mathrm{n}=3 ; \mathrm{P}<0.0001$ ). After another 3 days, the proliferation rate of the control was $3.34 \pm 0.01 \%$ of BrdU incorporation, compared with $5.7 \pm 0.12 \%$ for $\mathrm{Y} 27632$ treatment (Fig. 3B; $n=3 ; \mathrm{P}<0.0001$ ). In addition, the results revealed that BrdU-positive cells was also significantly increased following treatment with fasudil, another ROCK inhibitor, for 6 days (Fig. 3C; $\mathrm{P}<0.0001$ ). These findings indicated that treatment with ROCK inhibitors, particularly Y27652, resulted in a 2.5-fold increase in hiPSC-CM proliferation.

ROCK inhibitor inhibits the apoptosis of dissociated hiPSC-CMs in serum-free medium. To assess the potential effects of Y27632 and fasudil (both $10 \mu \mathrm{M}$ ) on hiPSC-CM apoptosis under serum starvation and suspension conditions, hiPSC-CMs suspended in serum-free media were seeded into non-adhesive dishes and cultured for 3 days. Apoptosis was then evaluated using Annexin V/PI staining. Following treatment with fasudil, number of apoptotic cells was significantly decreased compared with control ( $\mathrm{P}<0.0001$; Fig. 4A). 
A
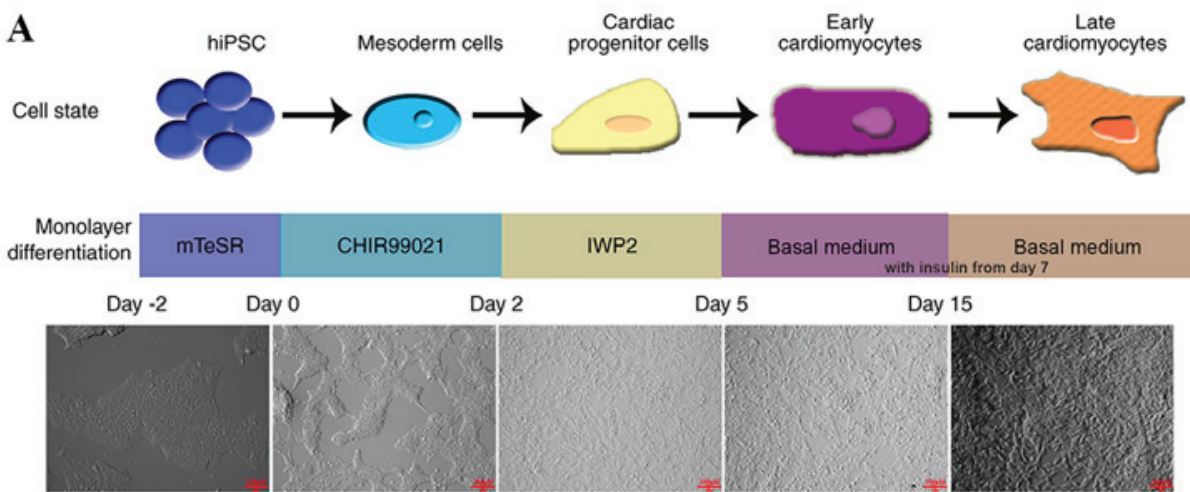

C
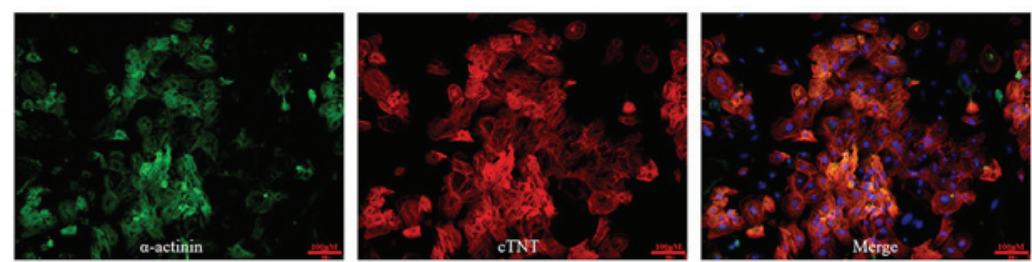

B

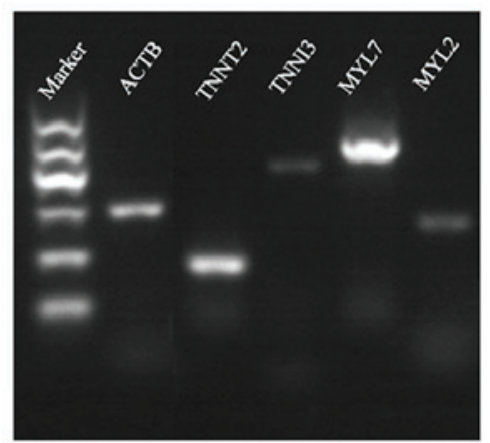

D

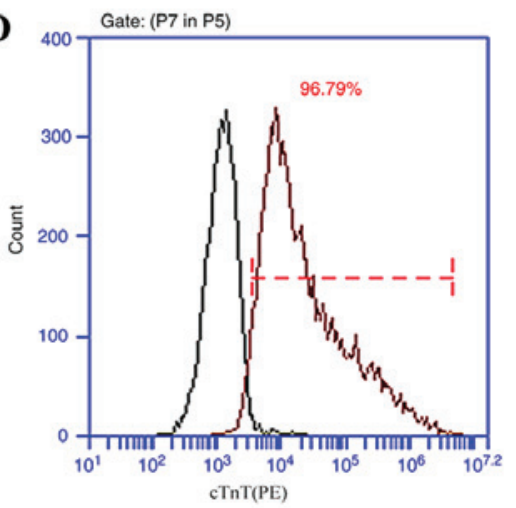

Figure 1. hiPSC differentiation into hiPSC-CM and characterization. (A) Schematic illustration of experimental pipeline applied for the differentiation of human iPSC into cardiomyocytes. The basal medium was supplemented with insulin from day 7 onwards. (B) Reverse transcription-PCR analysis revealed that cardiac gene expression was positive 30 days after the differentiation process commenced. (C) On day 15 , cells were purified and re-plated onto $0.1 \%$ (wt/vol) gelatin-coated coverslips. Immunostaining for $\alpha$-actinin (green) and cTnT (red) shows sarcomere organization. (Scale bar, $100 \mu \mathrm{m}$ ). (D) Representative dot plots for cTnT populations. Left peaks represent the isotype control. Numbers in plots indicate the percentage of cTnT-positive cells after day 21 of differentiation. hiPSC, human induced pluripotent stem cell; CM, cardiomyocites; ACTB, $\beta$-actin; ACTB, $\beta$-actin; TNNT2, troponin T type 2 ; TNNI3, troponin I type 3 ; MYL7, Myosin light chain 7; MYL2, Myosin light chain 2; hiPSC, human induced pluripotent stem cells; CM, cardiomyocte; cTnT, cardiac troponin T.

A

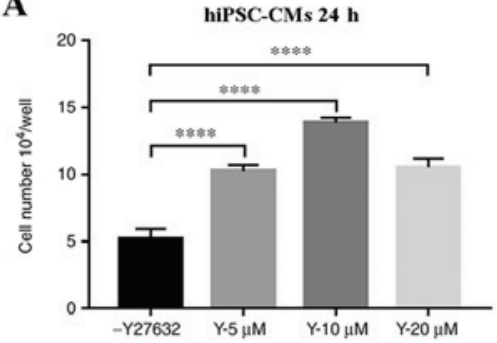

C

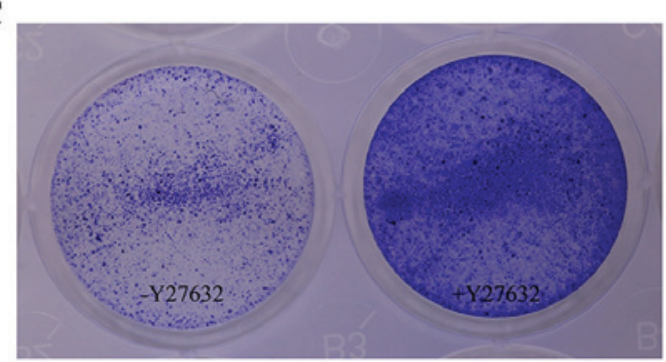

B
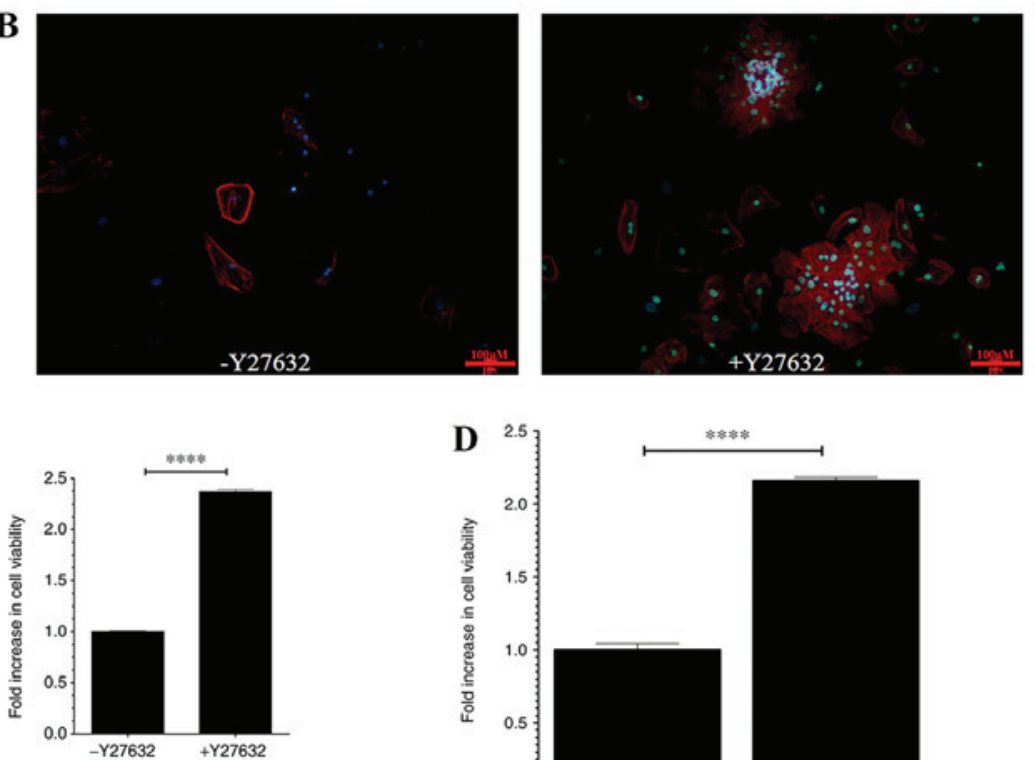

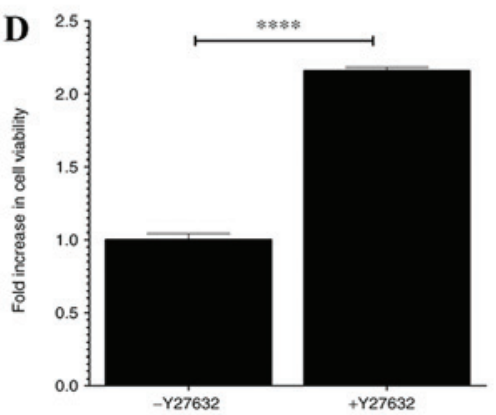

Figure 2. ROCK inhibitor markedly improved hiPSC-CM viability. (A) Y27632 promotes the numbers of surviving cells following passage by single-cell dissociation in a dose-dependent manner. ${ }^{* * * *} \mathrm{P}<0.0001$ ( $\mathrm{n}=3$ ). (B-D) Dissociated hiPSC-CMs were plated onto $0.1 \%$ (wt/vol) gelatin-coated dishes and treated with or without $10 \mu \mathrm{M}$ Y27632 for $24 \mathrm{~h}$. (B) Immunostaining of hiPSC-CM markers $24 \mathrm{~h}$ after $10 \mu \mathrm{M}$ Y27632 treatment. cTnT (red), DAPI (blue). Scale bar, $100 \mu \mathrm{m}$. (C) The total number of cells were quantified using crystal violet staining following $10 \mu \mathrm{M}$ Y27632 treatment. ${ }^{* * * * *} \mathrm{P}<0.0001$ (n=3). (D) Effect of $10 \mu \mathrm{M}$ Y27632 treatment on the viability of hiPSC-CMs was examined using a Cell Counting kit- 8 assay. ${ }^{* * * * *} \mathrm{P}<0.0001$. ROCK, Rho-associated kinase; hiPSC-CMs, human induced pluripotent stem cells-derived cardiomyocytes; cTnT, cardiac troponin T. 

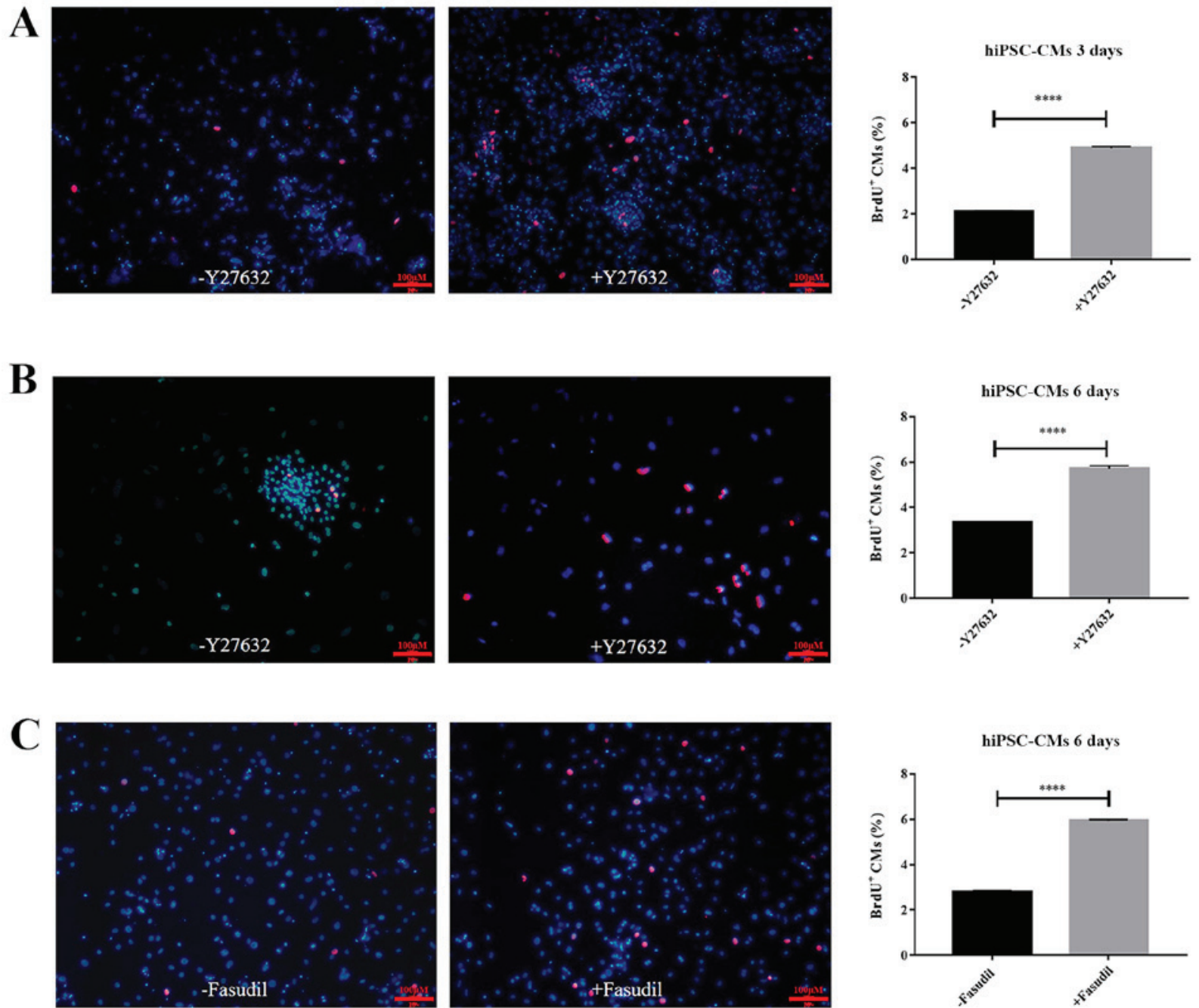

Figure 3. Effects of ROCK inhibitor on the proliferation of hiPSC-CMs, as reported using a BrdU assay. BrdU+ cells are presented in red and nuclei were stained in blue. (A) Cells were cultured with or without Y27632 for 3 and (B) 6 days. (C) Cells were treated with or without fasudil for 6 days. ${ }^{* * * * *} \mathrm{P}<0.0001$. ROCK, Rho-associated kinase; hiPSC-CMs, human induced pluripotent stem cells-derived cardiomyocytes; BrdU, 5-Bromo-2-deoxyUridine.

In addition, Y27632 treatment also significantly reduced cell apoptosis compared with control $(\mathrm{P}<0.001$; Fig. 4B). Therefore, treatment with ROCK inhibitors significantly reduced hiPSC-CM apoptosis and necrosis, increasing survival under conditions of stress.

To examine the potential mechanism of $\mathrm{Y} 27632$ on the inhibition of apoptosis, mRNA levels of Bcl-2, Bax, caspases-3 and -8 were detected after dissociated hiPSC-CMs were cultured in suspension in the presence or absence of Y27632 for $72 \mathrm{~h}$. The expression of caspase-3 and -8 were significantly reduced by $\mathrm{Y} 27632(\mathrm{P}<0.001)$, whilst levels of Bcl-2 and Bax did not exhibit significant differences (Fig. 4C and D).

Subsequent western blot analysis revealed that Y27632 significantly reduced the protein expression of cleaved caspase- 3 and caspase- 8 ( $\mathrm{P}<0.0001$; Fig. 4E). The colorimetric assay of caspase- 3 activity is based on the spectrophotometric detection of pNA, which is produced by caspase-3-mediated cleavage of acetyl-Asp-Glu-Val-Asp p-nitroanilide (Ac-DEVD-pNA). The level of caspase-3 activity demonstrated that treatment with Y27632 reduced caspase-3 activity $(\mathrm{P}<0.01$; Fig. 4F). These results suggested that the preventive effects of Y27632 on hiPSC-CMs apoptosis could be due to the suppression of caspase-3 activation.

\section{Discussion}

Since being generated from a culture of human fibroblasts using a cocktail of four transcription factors (Oct3/4, Sox 2, c-Myc and Klf4) in 2007, human iPSC technology has been widely used for disease modeling, drug discovery and the development of cell therapies $(18,19)$. Progress in hiPSC-derived cardiomyocytes has provided a platform for personalized cardiovascular medicine (20). The development of effective approaches in preventing cell death will facilitate the application of hiPSC-derived CMs in multiple research areas. In the present study, the effects of the ROCK inhibitor, Y27632, were evaluated in hiPSC-CMs.

Significant improvements have been made in the differentiation of pluripotent stem cells into cardiomyocytes over the past two decades, which progressed from an original efficiency of $5-10 \%$ to $>90 \%(20,21)$. In the present study, a directed cardiac differentiation method using small molecules (CHIR99021 and IWP2) that modulate Wnt signaling were 
A
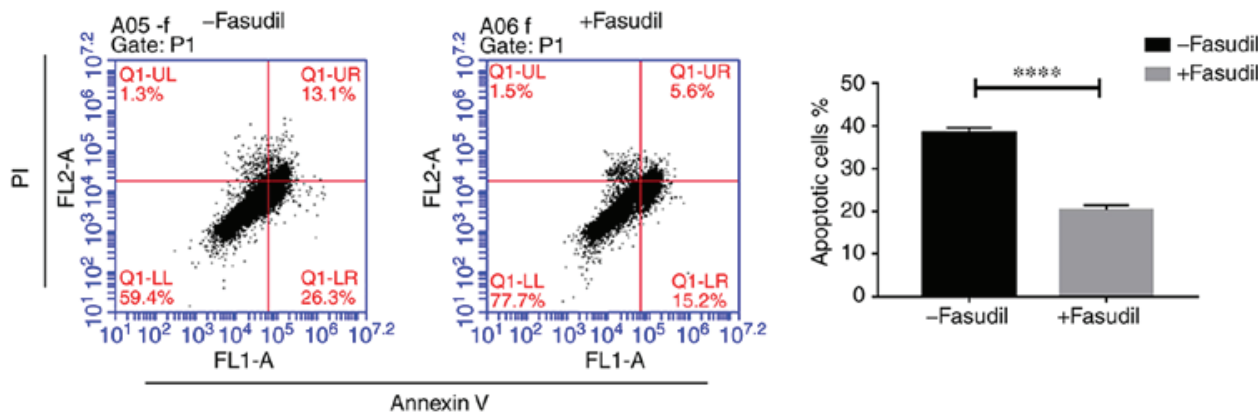

B
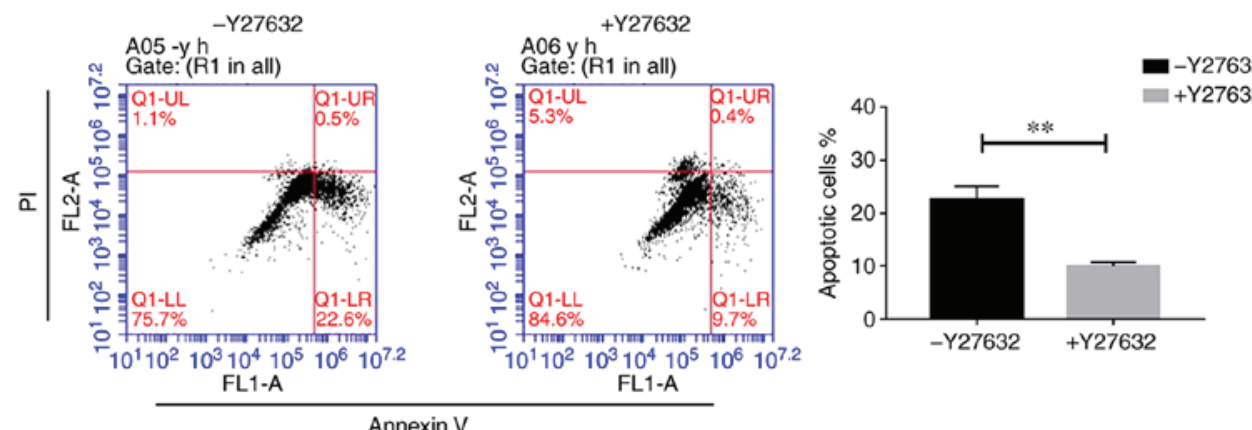

C

Annexin V

\section{D}
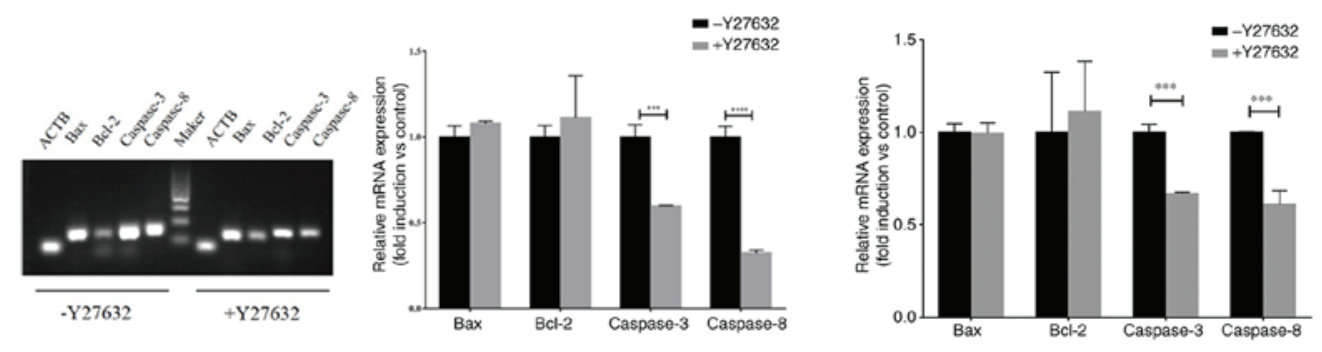

$\mathbf{E}$

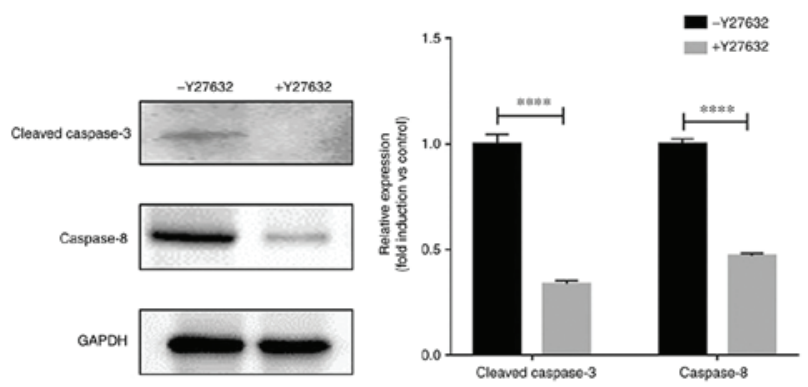

$\mathbf{F}$
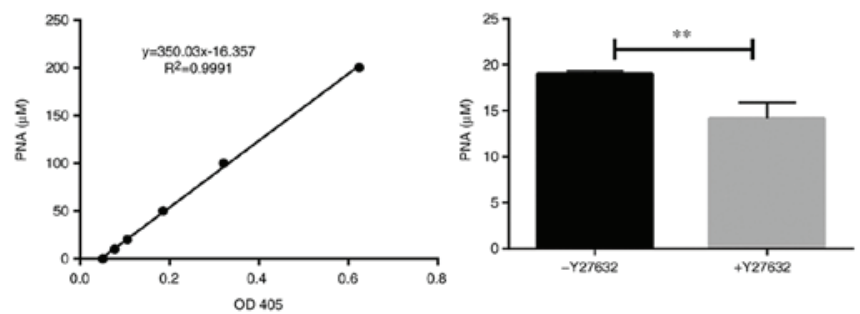

Figure 4. ROCK inhibitors prevent the apoptosis of dissociated hiPSC-CMs in serum-free medium. (A) Following treatment with or without fasudil for $72 \mathrm{~h}$, cells were collected and analyzed by flow cytometry using Annexin V-FITC/PI staining. ${ }^{* * * * *} \mathrm{P}<0.0001$. (B-E) hiPSC-CMs were cultured for 3 days in the presence or absence of Y27632. (B) Flow cytometric analysis were used to detect apoptosis. ${ }^{* *} \mathrm{P}<0.01$. (C) The expression of Bcl-2, Bax and caspases 3 and 8 were detected by reverse transcription-PCR and (D) quantitative PCR. ${ }^{* * *} \mathrm{P}<0.001$. (E) The expression of cleaved caspase-3 and caspase-8 were analyzed by western blotting, with GAPDH as the loading control. ${ }^{* * * *} \mathrm{P}<0.0001$. (F) Y27632 treatment reduced the activity of caspase-3 in detached hiPSC-CMs in suspension culture. ${ }^{* *} \mathrm{P}<0.01$. ROCK, Rho-associated kinase; hiPSC-CMs, human induced pluripotent stem cells-derived cardiomycytes; PI, propidium iodide; PNA, p-nitroaniline; OD, optical density. 


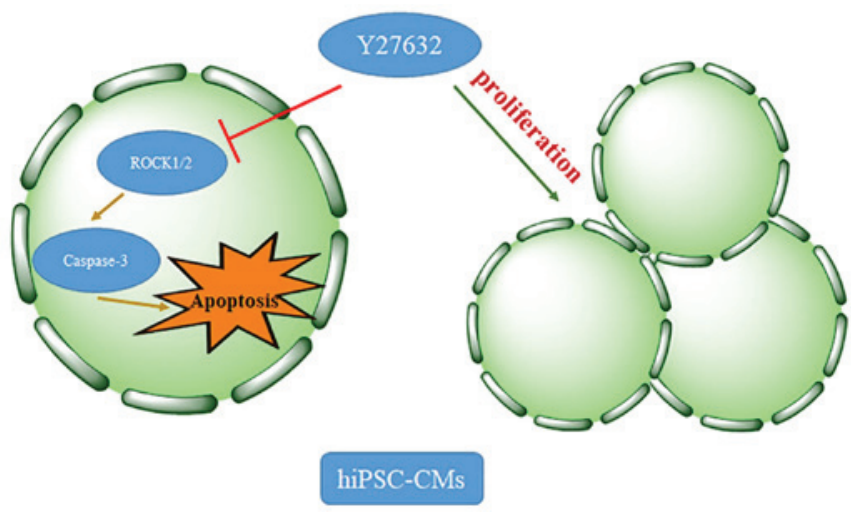

Figure 5. Summary of the effects of the ROCK inhibitor Y27632 on hiPSC-CM physiology as reported in the present study. ROCK, Rho-associated kinase; hiPSC-CMs, human induced pluripotent stem cells-derived cardiomyocytes.

applied, which achieved an efficiency of $\sim 96.79 \%$. Small molecules are advantageous compared with growth factors in that they are more cost effective, easy to store, more stable and more amenable to quality control (22).

Although structurally and functionally immature hiPSC-CM limits its application in drug development and cell therapy, their fetal characteristics may provide a possibility for hiPSC-CMs to proliferate (23). Uosaki et al (24) identified four chemical compound groups: Inhibitors of glycogen synthase kinase-3, p38 mitogen-activated protein kinase, $\mathrm{Ca}^{2+} /$ calmodulin-dependent protein kinase II and activators of extra cellular signal-regulated kinase, all of which synergistically enhanced the proliferation of cardiomyocytes derived from both mouse and human PSCs. The present study demonstrated that the ROCK inhibitor Y27632 exhibited proliferative effects on hiPSC-CMs. Recently, Mohamed et al (25) screened a list of cell-cycle regulators expressed in proliferating fetal cardiomyocytes. It was demonstrated that the overexpression of cyclin-dependent kinase (CDK) 1, CDK4, cyclin B1 and cyclin D1 efficiently induced cell division in the post-mitotic mouse, rat and hiPSC-derived cardiomyocytes (25). However, the potential relationship between ROCK inhibition and cell-cycle regulation requires further investigation.

Y27632 has been demonstrated to suppress cardiac cell apoptosis in vivo and in vitro. Previous studies have demonstrated that ROCK inhibitors enhanced post-ischemia cardiac function, suppressed cardiac cell apoptosis and decreased the accumulation of neutrophils in the heart following ischemia and reperfusion injury in mice (26-29). Bian et al (30) reported that the pretreatment of Y27632 and 3-aminobenzamide reduced myocardial infarction size and cardiomyocyte apoptosis via the poly (ADP-ribose) polymerase/ERK signaling pathway. More recently, Dong et al (31) evaluated the cardioprotective effects of Y27632 and demonstrated that Y27632 attenuated myocardial injury by inhibiting the activation of the MAPK and NF- $\kappa \mathrm{B}$ signaling pathway, suppressing apoptosis and the inflammatory response. The relationship between the Y27632-mediated inhibition of apoptosis in the present study with the MAPK and NF- $\kappa$ B signaling pathways merits further exploration.

ROCK inhibitors have been previously applied in tissue engineering to improve cardiac cell engraftment for a number of cardiovascular diseases based on cell therapy. Recently, Zhao et al (32) revealed that preconditioning with Y27632 increased the engraftment rate of transplanted hiPSC-CMs in a murine myocardial infarction (MI) model by a reduction in the number of apoptotic hiPSC-CMs but reported no changes in proliferation. In the present study, the suppressive effects of Y27632 on hiPSC-CM apoptosis was potentially caused by the suppression of expression and activity of caspase- 3 and caspase-8. The results of proliferation were not consistent with those of Zhao et al (32), presumably due to different sources of hiPSC and differential experimental conditions, including treatment time and culture environments.

Additionally, previous studies have suggested that ROCK kinase is involved in cell adhesion to the extracellular matrix by modulating integrin avidity, which exerts further effects on downstream physiological functions $(7,33,34)$. In particular, Martewicz et al (35) demonstrated the involvement of a mechanotransduction pathway, RhoA/ROCK, in the structural reorganization of hPSC-derived cardiomyocytes after adhesion. Treatment with Y27632 prevented the structural reorganization of the sarcoplasmic reticulum (SR) after attachment, modulating SERCA2 localization and promoting calcium cycling. They also suggested that SR structural reorganization was observed in hPSC-CMs derived from an embryonic bodies-based differentiation protocol that is distinct from monolayer-based differentiation protocols (35). Recently, Yan et al (36) demonstrated that the application of Y27632 promoted the gene expression of matrix metalloproteinases 2/3 and Notch-1 signaling to modulate Yes-associated protein-1 localization in the regulation of efficient patterning of cardiovascular spheroids following mesoderm formation from hPSC. Therefore, the possibility that ROCK kinase-associated integrin signaling and receptors may be associated with cardiomyocyte maturation, physiological function, proliferation and apoptosis, which has not been examined in depth in the present study, cannot be ruled out $(37,38)$.

In conclusion, the present study demonstrated that treatment with the ROCK inhibitor Y27632 significantly promoted the survival of dissociated hiPSC-CMs by increasing proliferation and attenuating apoptosis (Fig. 5). The application of Rho-kinase inhibitors will be beneficial for the culturing of CMs, which may facilitate the development of applications of hiPSC-derived CMs for multiple research areas. However, the associated mechanisms require further elucidation.

\section{Acknowledgements}

The authors would like to thank Dr Palecek (Department of Chemical and Biological Engineering, University of Wisconsin, Madison, WI, USA), for guidance with cardiac-directed differentiation. The authors would also like to acknowledge $\mathrm{Mr}$. Ahmed Salah (Department of Biochemistry and Molecular Biology, College of Life Science and Medicine, Zhejiang Sci-Tech University, Hangzhou, Zhejiang, China) for the critical evaluation of the manuscript and helpful comments.

\section{Funding}

The current study was supported the Enterprise Commissioned R\&D Project (grant no. 16040135-J). 


\section{Availability of data and materials}

The datasets used and/or analyzed during the present study are available from the corresponding author on reasonable request.

\section{Authors' contributions}

NQ and YW conceived and designed the experiments of the current study. MK and MJ performed the experiments. MK wrote the manuscript. HW and YY analyzed the data. All authors approved the final version of the manuscript.

\section{Ethics approval and consent to participate}

Not applicable.

\section{Patient consent for publication}

Not applicable.

\section{Competing interests}

The authors declare that they have no competing interests.

\section{References}

1. Ebert AD, Diecke S, Chen IY and Wu JC: Reprogramming and transdifferentiation for cardiovascular development and regenerative medicine: Where do we stand? EMBO Mol Med 7: 1090-1103, 2015

2. Hartman ME, Dai DF and Laflamme MA: Human pluripotent stem cells: Prospects and challenges as a source of cardiomyocytes for in vitro modeling and cell-based cardiac repair. Adv Drug Deliv Rev 96: 3-17, 2016.

3. Kehat I, Kenyagin-Karsenti D, Snir M, Segev H, Amit M, Gepstein A, Livne E, Binah O, Itskovitz-Eldor J and Gepstein L: Human embryonic stem cells can differentiate into myocytes with structural and functional properties of cardiomyocytes. J Clin Invest 108: 407-414, 2001

4. Burridge PW, Matsa E, Shukla P, Lin ZC, Churko JM, Ebert AD, Lan F, Diecke S, Huber B, Mordwinkin NM, et al: Chemically defined generation of human cardiomyocytes. Nat Methods 11: 855-860, 2014.

5. Lian X, Zhang J, Azarin SM, Zhu K, Hazeltine LB, Bao X, Hsiao C, Kamp TJ and Palecek SP: Directed cardiomyocyte differentiation from human pluripotent stem cells by modulating Wnt/ $\beta$-catenin signaling under fully defined conditions. Nat Protoc 8: 162-175, 2013

6. Shimizu T and Liao JK: Rho kinases and cardiac remodeling. Circ J 80: 1491-1498, 2016.

7. Narumiya $S$ and Thumkeo D: Rho signaling research: History, current status and future directions. FEBS Lett 592: 1763-1776, 2018.

8. Shimizu T, Narang N, Chen P, Yu B, Knapp M, Janardanan J, Blair J and Liao JK: Fibroblast deletion of ROCK2 attenuates cardiac hypertrophy, fibrosis, and diastolic dysfunction. JCI Insight 2: pii: 93187, 2017.

9. Surma M, Wei L and Shi J: Rho kinase as a therapeutic target in cardiovascular disease. Future Cardiol 7: 657-671, 2011

10. Shibuya M and Suzuki Y: Treatment of cerebral vasospasm by a protein kinase inhibitor AT 877. No To Shinkei 45: 819-824, 1993 (In Japanese).

11. Chang J, Xie M, Shah VR, Schneider MD, Entman ML, Wei L and Schwartz RJ: Activation of Rho-associated coiled-coil protein kinase 1 (ROCK-1) by caspase-3 cleavage plays an essential role in cardiac myocyte apoptosis. Proc Natl Acad Sci USA 103: 14495-14500, 2006.

12. Hartmann S, Ridley AJ and Lutz S: The function of Rho-associated kinases ROCK 1 and ROCK2 in the pathogenesis of cardiovascular disease. Front Pharmacol 6: 276, 2015 .
13. Dong M, Ding W, Liao Y, Liu Y, Yan D, Zhang Y, Wang R, Zheng N, Liu S and Liu J: Polydatin prevents hypertrophy in phenylephrine induced neonatal mouse cardiomyocytes and pressure-overload mouse models. Eur J Pharmacol 746: 186-197, 2015.

14. Sawada N and Liao JK: Rho/Rho-associated coiled-coil forming kinase pathway as therapeutic targets for statins in atherosclerosis. Antioxid Redox Signal 20: 1251-1267, 2014.

15. Tohyama S, Hattori F, Sano M, Hishiki T, Nagahata Y, Matsuura T, Hashimoto H, Suzuki T, Yamashita H and Satoh Y: Distinct metabolic flow enables large-scale purification of mouse and human pluripotent stem cell-derived cardiomyocytes. Cell Stem Cell 12: 127-137, 2013.

16. Huang L, Li Q, Li H, He Z, Cheng Z, Chen J and Guo L: Inhibition of intracellular $\mathrm{Ca}^{2+}$ release by a Rho-kinase inhibitor for the treatment of ischemic damage in primary cultured rat hippocampal neurons. Eur J Pharmacol 602: 238-244, 2009.

17. Livak KJ and Schmittgen TD: Analysis of relative gene expression data using real-time quantitative PCR and the 2(-Delta Delta C(T)) method. Methods 25: 402-408, 2001.

18. Takahashi K, Tanabe K, Ohnuki M, Narita M, Ichisaka T, Tomoda K and Yamanaka S: Induction of pluripotent stem cells from adult human fibroblasts by defined factors. Cell 131: 861-872, 2007.

19. Yu J, Vodyanik MA, Smuga-Otto K, Antosiewicz-Bourget J Frane JL, Tian S, Nie J, Jonsdottir GA, Ruotti V, Stewart R, et al: Induced pluripotent stem cell lines derived from human somatic cells. Science 318: 1917-1920, 2007.

20. Matsa E, Ahrens JH and Wu JC: Human induced pluripotent stem cells as a platform for personalized and precision cardiovascular medicine. Physiol Rev 96: 1093-1126, 2016.

21. Qiu XX, Liu Y, Zhang YF, Guan YN, Jia QQ, Wang C, Liang H, Li YQ, Yang HT and Qin YW: Rapamycin and CHIR99021 coordinate robust cardiomyocyte differentiation from human pluripotent stem cells via reducing p53-dependent apoptosis. J Am Heart Assoc 6: pii: e005295, 2017.

22. Kim WH, Jung DW and Williams DR: Making cardiomyocytes with your chemistry set: Small molecule-induced cardiogenesis in somatic cells. World J Cardiol 7: 125-133, 2015.

23. Zhu R, Blazeski A, Poon E, Costa KD, Tung L and Boheler KR: Physical developmental cues for the maturation of human pluripotent stem cell-derived cardiomyocytes. Stem Cell Res Ther 5: 117, 2014.

24. Uosaki H, Magadum A, Seo K, Fukushima H, Takeuchi A, Nakagawa Y, Moyes KW, Narazaki G, Kuwahara K, Laflamme M, et al: Identification of chemicals inducing cardiomyocyte proliferation in developmental stage-specific manner with pluripotent stem cells. Circ Cardiovasc Genet 6: 624-633, 2013.

25. Mohamed TMA, Ang YS, Radzinsky E, Zhou P, Huang Y, Elfenbein A, Foley A, Magnitsky S and Srivastava D: Regulation of cell cycle to stimulate adult cardiomyocyte proliferation and cardiac regeneration. Cell 173: 104-116, 2018.

26. Bao W, Hu E, Tao L, Boyce R, Mirabile R, Thudium DT, Ma XL, Willette RN and Yue TL: Inhibition of Rho-kinase protects the heart against ischemia/reperfusion injury. Cardiovasc Res 61: 548-558, 2004

27. Dong M, Yan BP, Liao JK, Lam YY, Yip GW and Yu CM: Rho-kinase inhibition: A novel therapeutic target for the treatment of cardiovascular diseases. Drug Discov Today 15: 622-629, 2010.

28. Feng Y, LoGrasso PV, Defert O and Li R: Rho kinase (ROCK) inhibitors and their therapeutic potential. J Med Chem 59: 2269-2300, 2016.

29. Li Y, Zhu W, Tao J, Xin P, Liu M, Li J and Wei M: Fasudil protects the heart against ischemia-reperfusion injury by attenuating endoplasmic reticulum stress and modulating SERCA activity: The differential role for PI3K/Akt and JAK2/STAT3 signaling pathways. PLoS One 7: e48115, 2012.

30. Bian H, Zhou Y, Yu B, Shang D, Liu F, Li B and Qi J: Rho-kinase signaling pathway promotes the expression of PARP to accelerate cardiomyocyte apoptosis in ischemia/reperfusion. Mol Med Rep 16: 2002-2008, 2017.

31. Dong LY, Qiu XX, Zhuang Y and Xue S: Y-27632, a Rho-kinase inhibitor, attenuates myocardial ischemia-reperfusion injury in rats. Int J Mol Med 43: 1911-1919, 2019.

32. Zhao M, Fan C, Ernst PJ, Tang Y, Zhu H, Mattapally S, Oduk Y, Borovjagin AV, Zhou L, Zhang J and Zhu W: Y-27632 preconditioning enhances transplantation of human-induced pluripotent stem cell-derived cardiomyocytes in myocardial infarction mice. Cardiovasc Res 115: 343-356, 2019. 
33. Martinez-Rico C, Pincet F, Thiery JP and Dufour S: Integrins stimulate E-cadherin-mediated intercellular adhesion by regulating Src-kinase activation and actomyosin contractility. J Cell Sci 123: 712-722, 2010

34. Thuveson M, Gaengel K, Collu GM, Chin ML, Singh J and Mlodzik M: Integrins are required for synchronous ommatidial rotation in the Drosophila eye linking planar cell polarity signalling to the extracellular matrix. Open Biol 9: 190148, 2019.

35. Martewicz S, Serena E, Zatti S, Keller G and Elvassore N: Substrate and mechanotransduction influence SERCA2a localization in human pluripotent stem cell-derived cardiomyocytes affecting functional performance. Stem Cell Res 25: 107-114, 2017.

36. Yan Y, Bejoy J, Xia J, Griffin K, Guan J and Li Y: Cell population balance of cardiovascular spheroids derived from human induced pluripotent stem cells. Sci Rep 9: 1295, 2019.
37. Manzur MJ, Aguilera MO, Kotler ML, Berón W and Ciuffo GM: Focal adhesion kinase, RhoA, and p38 mitogen-activated protein kinase modulates apoptosis mediated by angiotensin II $\mathrm{AT}_{2}$ receptors. J Cell Biochem 120: 1835-1849, 2019.

38. Vitillo L, Baxter M, Iskender B, Whiting P and Kimber SJ: Integrin-associated focal adhesion kinase protects human embryonic stem cells from apoptosis, detachment, and differentiation. Stem Cell Reports 7: 167-176, 2016.

This work is licensed under a Creative Commons Attribution-NonCommercial-NoDerivatives 4.0 International (CC BY-NC-ND 4.0) License. 